Citation: Unal, I., "Skew Semi-invariant Submanifolds of Generalized Kenmotsu Manifolds". Journal of Engineering Technology and Applied Sciences 5 (3) 2020 : 103-110.

\title{
SKEW SEMI-INVARIANT SUBMANIFOLDS OF GENERALIZED KENMOTSU MANIFOLDS
}

\author{
Inan Unal \\ Department of Computer Engineering, Munzur University, Tunceli, Turkey \\ inanunal@munzur.edu.tr
}

\begin{abstract}
In this paper, we study a special submanifold of a generalized Kenmotsu manifold. Firstly, we define a skew semi-invariant submanifold of a generalized Kenmotsu manifold and give an example. Later, we obtain some basic results for such submanifolds. Finally, we investigate the geometry of distributions.
\end{abstract}

Keywords: Semi-invariant submanifold, skew semi-invariant submanifold, generalized Kenmotsu manifold

\section{Introduction}

In 1972 K.Kenmotsu [1] defined a new type of contact manifolds. This type of manifolds known as Kenmotsu manifolds and they are an important class of contact manifolds. There are many geometric properties different from Sasakian manifolds. For example, although a Kenmotsu manifold is normal, it is not Sasakian. Also, a Kenmotsu manifold is not compact and it has negative sectional curvature. For a cemperensive introduction to Kemotsu manifolds, we refer to reader [2]. M. Falcitelli and A. M. Pastore [3] introduced Kenmotsu f.pk -manifold which has an $f$-structure. In [4], Turgut Vanlı and Sarı defined and studied generalized Kenmotsu manifold (GK-manifold). GK-manifolds have been studied in [4-8].

Submanifold theory is a major notion in the contact geometry. We classify submanifolds of contact manifolds such as submanifolds of complex manifolds. One kind of submanifolds is CR-submanifold which is defined and studied by A. Bejancu [9] for a Kaehlerian manifold. By the following works, this subject has been carried to contact manifolds. Some different classes of submanifolds which have been defined for almost contact structures, are semi-invariant, invariant, anti-invariant etc. Almost semi-invariant submanifolds of a Sasakian manifold was studied by Bejancu and Papaghuic [10]. They also gave a classification for submanifold classes of a Sasakian manifold. Except for these submanifold classes also we have slant submanifolds 
of complex and contact manifolds. Ronsse [11] defined the notion of skew CR-submanifolds of Kaehler manifolds. Also, we have the notion skew semi-invariant submanifolds for contact manifolds. Many researchers studied on this subject in [12-15].

In this study, we work on skew semi-invariant submanifolds of GK-manifolds. After present fundamental facts on GK-manifolds and submanifold theory, we define skew semi-invariant submanifolds of GK-manifolds. Then, we obtain some basic properties of such manifolds. Finally, we examine the geometry of distributions.

\section{Preliminaries}

In this section we present some fundamental facts on GK-manifolds. For detail we refer to reader [4]. Also, we give basic tools of the submanifold theory.

Definition 2.1 A $(2 n+s)$-dimensional differentiable manifold $\bar{M}$ is called metric $f$ manifold if there exist an $(1,1)$ type tensor field $\varphi, s$ vector fields $\xi_{1}, \ldots, \xi_{s}, s$-forms $\eta^{1}, \ldots, \eta^{s}$ and a Riemannian metric $g$ on $\bar{M}$ such that

$\varphi^{2}=-I+\sum_{i=1}^{s} \eta^{i} \otimes \xi_{i}$

$\eta^{i}\left(\xi_{j}\right)=\delta_{i j}, \varphi \xi_{i}=0, \eta^{i} \circ \varphi=0$

$g(\varphi X, \varphi Y)=g(X, Y)-\sum_{i=1}^{s} \eta^{i}(X) \eta^{i}(Y)$

$\eta^{i}(X)=g\left(X, \xi_{i}\right), g(X, \varphi Y)=-g(\varphi X, Y)$,

for any $X, Y \in \Gamma(T \bar{M}), i, j \in\{1, \ldots, s\}[16]$.

Second fundamental 2 -form $\Phi$ is defined by $\Phi(X, Y)=g(X, \varphi Y)$, for any $X, Y \in \Gamma(T \bar{M})$. If we denote $\mathcal{L}=\left\{X: \eta^{\alpha}(X)=0,1 \leq \alpha \leq s\right\}$ and $\mathcal{M}=s p\left\{\xi_{1}, \xi_{2}, \ldots, \xi_{s}\right\}$ then we have following direct sum $: T \bar{M}=\mathcal{L} \oplus \mathcal{M}$.

A metric $f$-manifold is normal if $[\varphi, \varphi]+2 \sum_{i=1}^{s} d \eta^{i} \otimes \xi_{i}=0$, where $[\varphi, \varphi]$ is the Nijenhuis tensor of $\varphi$. For a 2 -form $\Phi$ on $\bar{M}$ such that $\eta^{1} \wedge \ldots \wedge \eta^{s} \wedge \Phi^{n} \neq 0, M$ is called an almost scontact metric manifold. If $M$ is also normal, then it is called an s-contact metric manifold.

Definition 2.2 Let $\bar{M}$ be an almost $s$ - contact metric manifold of dimension $(2 n+s), s \geq 1$, with $\left(\varphi, \xi_{i}, \eta^{i}, g\right) . \bar{M}$ is said to be a generalized almost Kenmotsu manifold if for all $1 \leq i \leq s$, 1 - forms $\eta^{i}$ are closed and $d \Phi=2 \sum_{i=1}^{s} \eta^{i} \wedge \Phi$. A normal generalized almost Kenmotsu manifold $\bar{M}$ is called a generalized Kenmotsu manifold (GK-manifold) [4].

By the following theorem we characterize the $s$-contact metric manifolds as GK-manifold. 
Theorem 2.3 An almost $s$-contact metric manifold $\left(\bar{M}, \varphi, \xi_{i}, \eta^{i}, g\right)$ is a GK-manifold if and only if

$$
\left(\bar{\nabla}_{X} \varphi\right) Y=\sum_{i=1}^{s}\left\{g(\varphi X, Y) \xi_{i}-\eta^{i}(Y) \varphi X\right\}
$$

for all $X, Y \in \Gamma(T \bar{M}), i \in\{1,2, \ldots, s\}$, where $\bar{\nabla}$ is Riemannian connection on $\mathrm{M}$ [4].

Let $\bar{M}$ be $(2 n+s)$-dimensional a GK- manifold with structure $\left(\varphi, \xi_{i}, \eta^{i}, g\right)$. Then we have $\bar{\nabla}_{X} \xi_{i}=-\varphi^{2} X$ for all $X \in \Gamma(T \bar{M})[4]$.

Let $M$ be an m-dimensional submanifold isometrically immersed in a GK-manifold $\bar{M}$. Denote by $T M$ and $T M^{\perp}$ the tangent bundle and the normal bundle of $M$, respectively. Suppose that the structure vectors field $\xi_{i}$ be tangent to the submanifold $M$, and let denote $\{\bar{\xi}\}$ as $s$-dimensional distribution spanned by $\left\{\xi_{1}, \ldots, \xi_{s}\right\}$ on $M$ and $\{\bar{\xi}\}^{\perp}$ be orthogonal complementary of $\{\bar{\xi}\}$ in $T M$.

For any $X \in \Gamma(T M)$ we have $g\left(\varphi X, \xi_{i}\right)=0$. Then, we put

$$
\varphi X=b X+c X
$$

for $b X \in \Gamma(T M)$ and $c X \in \Gamma\left(T M^{\perp}\right)$, where $b$ is an endomorphism of the tangent bundle $T M$ and $c$ is a normal bundle valued 1-form on $M$. On the other hand, for each $x \in M$ we define the following subspaces of $T M$.

$$
D_{x}=\left\{X_{x} \in\{\bar{\xi}\}_{x}^{\perp}: c\left(X_{x}\right)=0\right\}
$$

and

$D_{x}^{\perp}=\left\{X_{x} \in\{\bar{\xi}\}_{x}^{\perp}: b\left(X_{x}\right)=0\right\}$.

We note that $D_{x}$ and $D_{x}^{\perp}$ are two orthogonal subspaces of the tangent space $T_{x} M$ [10]. In fact, using (2.1), (2.4) and (2.6), we have $g(X, Y)=g(\varphi X, \varphi Y)=g(b X, c Y)=0$ for any $X_{x} \in D_{x}$ and $Y_{x} \in D_{x}^{\perp}$.

Definition 2.4 A submanifold $M$ of the GK-manifold $\bar{M}$ is said to be a skew semi-invariant submanifold if its tangent bundle $T M$ has the decomposition $T M=D \oplus D^{\perp} \oplus \tilde{D} \oplus\{\bar{\xi}\}$ such as

i. $\{\bar{\xi}\}$ is distribution spanned on $M$ by the vector field $\left\{\xi_{1}, \ldots, \xi_{s}\right\}$

ii. $\quad D$ is an invariant distribution on $M$, that is $\varphi\left(D_{x}\right)=D_{x}$ for each $x \in M$

iii. $\quad D^{\perp}$ is an anti invariant distribution on $M$, that is, $\varphi\left(D_{x}^{\perp}\right) \subset T_{x} M^{\perp}$, for each $x \in M$

iv. $D$ is neither an invariant nor an anti invariant distribution on $M$ that is $b X_{x} \neq 0$ and $c X_{x} \neq 0$ for any $x \in M$ and $X_{x} \in D_{x}$. 
From the definition, we have following classification:

A skew semi-invariant submanifold $M$ in a GK-manifold $\bar{M}$ is said to be,

1. a semi-invariant submanifold if we have $D=\{0\}$

2. a semi-slant submanifold if we have $D^{\perp}=\{0\}$

3. a hemi-slant submanifold if we have $D=\{0\}$

4. an invariant submanifold if we have $D^{\perp}=\{0\}$ and $D=\{0\}$

5. an anti invariant submanifold if we have $D=\{0\}$ and $D=\{0\}$

6. a slant-submanifold if we have $D=\{0\}$ and $D^{\perp}=\{0\}$.

In the following example we give a skew semi-invariant submanifold of a canonical GKmanifold's example $\mathbb{R}^{2 n+s}$.

Example 2.5 Let take a usual GK-structure $\left(\varphi, \eta^{j}, \xi_{j}, g\right)$ on $\mathbb{R}^{2 n+s}$ such as

$\eta^{j}=d z_{j}, \quad \xi_{i}=\frac{\partial}{\partial z_{i}}$

$\varphi\left(\sum_{i=1}^{n}\left(X_{i} \frac{\partial}{\partial x_{i}}+Y_{i} \frac{\partial}{\partial y_{i}}\right)+\sum_{j=1}^{s} Z_{j} \frac{\partial}{\partial z_{j}}\right)=\sum_{i=1}^{n}\left(Y_{i} \frac{\partial}{\partial x_{i}}-X_{i} \frac{\partial}{\partial y_{i}}\right)+\sum_{i=1}^{n} \sum_{j=1}^{s} Y_{i} \frac{\partial}{\partial z_{j}}$

$g=e^{-2 z}\left(\sum_{i=1}^{n} d x_{i} \otimes d x_{i}+d y_{i} \otimes d y_{i}\right)-\sum_{j=1}^{s} \eta^{j} \otimes \eta^{j}$

$x_{1}, \ldots, x_{n}, y_{1}, \ldots, y_{n}, z_{1}, \ldots, z_{s}$ denoting the cartesian coordinates on $\mathbb{R}^{2 n+1}$.

Let consider a submanifold of $\mathbb{R}^{10}$ defined by

$M=X\left(u, v, k, l, s, w, t_{1}, t_{2}\right)=\left(u, 0, k, s, v, l, \cos w, \sin w, t_{1}, t_{2}\right)$.

Then the local frame of $T M$ is given by

$e_{1}=\frac{\partial}{\partial x_{1}}, e_{2}=\frac{\partial}{\partial y_{1}}, e_{3}=\frac{\partial}{\partial x_{3}}, e_{4}=\frac{\partial}{\partial y_{2}}, e_{5}=\frac{\partial}{\partial x_{4}}$,

$e_{6}=-\sin w \frac{\partial}{\partial y_{3}}+\cos w \frac{\partial}{\partial y_{4}}, e_{7}=\frac{\partial}{\partial z_{1}}=\xi_{1}, e_{8}=\frac{\partial}{\partial z_{2}}=\xi_{2}$

and $e_{1}^{*}=\frac{\partial}{\partial x_{2}}, e_{2}^{*}=\frac{\partial}{\partial y_{3}}$.

Thus, we determine $D=s p\left\{e_{1}, e_{2}\right\}, D^{\perp}=s p\left\{e_{3}, e_{4}\right\}$, and $D=s p\left\{e_{5}, e_{6}\right\}$. Then $D, D^{\perp}$ and $D$ become invariant, anti-invariant and neither an invariant nor an anti-invariant distribution, respectively. Thus $T M=D \oplus D^{\perp} \oplus D \oplus s p\left\{\xi_{1}, \xi_{2}\right\}$ is a skew semi-invariant submanifold of $\mathbb{R}^{10}$

\section{Basic properties of skew semi-invariant submanifolds of GK-manifold}

Let $M$ be a skew semi-invariant submanifold in a GK-manifold $\bar{M}$. The projection morphisms of $M$ to distributions $D, D^{\perp}$ and $D$ are denoted by $P, Q$ and $L$, respectively. Then for each $X \in \Gamma(T M)$ we can write 
$X=P X+Q X+L X+\eta^{i}(X) \xi_{i}$

For non-zero vector field $X \in \Gamma(D)$ we note that $b X \neq 0$ and $c X \neq 0$. Thus $c$ defines a vector subbundle $c D: x \rightarrow c D_{x}$ of $T M^{\perp}$. Also, we have $g\left(\varphi D^{\perp}, c D\right)=0$. For any $N \in \Gamma\left(T M^{\perp}\right)$ we put

$\varphi N=t N+f N$

where $t N$ and $f N$ are the tangential and normal components of $\varphi N$, respectively. Next, we denote by $\mu$ the orthogonal complementary vector bundle to $\varphi D^{\perp} \oplus c D$ in $T M^{\perp}$. Thus we have that the normal bundle to $\mathrm{M}$ has the decomposition $T M^{\perp}=\varphi D^{\perp} \oplus c D \oplus \mu$. The Gauss equation is given by

$\bar{\nabla}_{X} Y=\nabla_{X} Y+h(X, Y)$

for any $X, Y \in \Gamma(T M)$ and $N \in \Gamma(T M)^{\perp}$, where $\bar{\nabla}$ and $\nabla$ Levi-Civita connection on $M$ and induced connection on $M$, respectively.

The Weingarten equation is given by

$\bar{\nabla}_{X} N=-A_{N} X+\nabla_{X}^{\perp} N$

for any $X \in \Gamma(T M)$ and $N \in \Gamma\left(T M^{\perp}\right)$, where $\nabla^{\perp}$ is the connection in the normal bundle, $h$ is the second fundamental from of $M$. The second fundamental form $h$ and the shape operator $A$ have a relation as $g(h(X, Y), V)=g\left(A_{V} X, Y\right)$. By the following lemmas we obtain some basic relations on components of vector fields on $M$. The proofs could be obtained from Gauss and Weingarten formulas with using (3.1) .

Lemma 3.1 Let $M$ be a skew semi-invariant submanifold of a GK-manifold $\bar{M}$. Then;

1. The decompositions of the vector fields on $M$ is given by

$$
\begin{aligned}
& P \nabla_{X} b Y=\varphi P\left(\nabla_{X} Y\right)+P A_{c Y} X-\eta(Y) P X, \\
& Q \nabla_{X} b Y=Q A_{c Y} X+Q \operatorname{th}(X, Y)-\eta(Y) Q X, \\
& L \nabla_{X} b Y=b L\left(\nabla_{X} Y\right)+L A_{c Y} X+L t h(X, Y)-\eta(Y) L X, \\
& \eta\left(\nabla_{X} b Y\right)=\eta\left(A_{c Y} X\right)+g(\varphi X, \varphi Y), \\
& h(X, b Y)+\nabla_{X}^{\perp} c Y=c \nabla_{X} Y+f h(X, Y), \\
& \text { for all } X, Y \in \Gamma(T M) .
\end{aligned}
$$

2. The covariant derivation of vector fields in distributions are given by

$$
\begin{aligned}
& \nabla_{X} \xi_{i}=P X \text { and } h\left(X, \xi_{i}\right)=0, \text { for any } X \in \Gamma(D), \\
& \nabla_{Y} \xi_{i}=0 \text { and } h\left(Y, \xi_{i}\right)=-\varphi^{2} Y, \text { for any } Y \in \Gamma\left(D^{\perp}\right), \\
& \nabla_{Z} \xi_{i}=0 \text { and } h\left(Z, \xi_{i}\right)=-\varphi c L Z, \text { for any } Z \in \Gamma(D)
\end{aligned}
$$




$$
\nabla_{\xi_{i}} \xi_{j}=0 \text { and } h\left(\xi_{i}, \xi_{j}\right)=0
$$

3. The covariant derivation of vector fields on the direction of $\xi_{i}$ are given by

$$
\begin{aligned}
& \nabla_{\xi_{i}} U \in \Gamma(D) \text { for any } U \in \Gamma(D), \\
& \nabla_{\xi_{i}} V \in \Gamma\left(D^{\perp}\right) \text { for any } V \in \Gamma\left(D^{\perp}\right), \\
& \nabla_{\xi_{i}} W \in \Gamma(D) \text { for any } W \in \Gamma(D) .
\end{aligned}
$$

4. The Lie derivations of any vector fields are given by

$$
\begin{aligned}
& {\left[X, \xi_{i}\right] \in \Gamma(D) \text { for any } X \in \Gamma(D),} \\
& {\left[Y, \xi_{i}\right] \in \Gamma(D) \text { for any } Y \in \Gamma\left(D^{\perp}\right),} \\
& {\left[Z, \xi_{i}\right] \in \Gamma(D) \text { for any } Z \in \Gamma(D) .}
\end{aligned}
$$

\section{The geometric properties of distributions}

In this section, we examine that the distributions $D, D^{\perp}, D, D \oplus\{\bar{\xi}\}$ are involutive or not.

Theorem 4.1 Let $M$ be a skew semi-invariant submanifold of a GK-manifold $\bar{M}$. Then the distribution $D$ is not involutive.

Proof. For all $X, Y \in \Gamma(D)$, we have $g\left(X, \xi_{i}\right)=0$. Then, we get $g\left(\nabla_{Y} X, \xi_{i}\right)=g\left(\nabla_{Y} \xi_{i}, X\right)$. Thus, we obtain $g\left([X, Y], \xi_{i}\right)=g\left(\nabla_{X} \xi_{i}, Y\right)-g\left(\nabla_{Y} \xi_{i}, X\right)$. By using (3.5), we get $g\left([X, Y], \xi_{i}\right)=2 g(X, P Y)$ which completes the proof.

Theorem 4.2 Let $M$ be a skew semi-invariant submanifold of a GK-manifold $\bar{M}$. Then the distribution $D^{\perp}$ is always involutive.

Proof. For all $U, V \in \Gamma\left(D^{\perp}\right)$ and by using (3.6) we obtain $g\left([U, V], \xi_{i}\right)=g\left(\nabla_{U} \xi_{i}, V\right)-g\left(\nabla_{V} \xi_{i}, U\right)=0$. This shows us $D^{\perp}$ is involutive.

Theorem 4.3 Let $M$ be a skew semi-invariant submanifold of a GK-manifold $\bar{M}$. Then, the distribution $D$ is always involutive.

Proof. For all $K, W \in \Gamma(D)$, by using (3.7) we have $g\left([K, W], \xi_{i}\right)=g(\varphi L K, b L W)-g(\varphi L W, b L K)$. It is seen from here that $D$ is involutive.

Theorem 4.4 Let $M$ be a skew semi-invariant submanifold of a GK-manifold $\bar{M}$. The distribution $D \oplus \bar{\xi}$ is involutive if and only if $h(U, \varphi V)=h(U, \varphi V)$ for all $U, V \in \Gamma(D)$.

Proof. For all $U, V \in \Gamma(D \oplus \bar{\xi})$, we get $\varphi([U, V])=\bar{\nabla}_{U} \varphi V-\left(\bar{\nabla}_{U} \varphi\right) V-\bar{\nabla}_{V} \varphi U+\left(\bar{\nabla}_{V} \varphi\right) U$. Then, using (2.5) and (3.3) we have 


$$
\begin{aligned}
\varphi([U, V]) & =\nabla_{U} \varphi V+h(U, \varphi V)-\sum_{i=1}^{s}\left\{g(\varphi U, V) \xi_{i}-\eta^{i}(U) \varphi V\right\} \\
& -\nabla_{V} \varphi U-h(V, \varphi U)+\sum_{i=1}^{s}\left\{g(\varphi V, U) \xi_{i}-\eta^{i}(V) \varphi U\right\} .
\end{aligned}
$$

Then we obtain that $[U, V] \in \Gamma(D \oplus \bar{\xi})$ if and only if $h(U, \varphi V)=h(V, \varphi U)$ where $\varphi([U, V])$ is the component of $\nabla_{U} V$ from orthogonal complementary distribution of $D \oplus \bar{\xi}$.

Corollary 4.5 Let $M$ be a skew semi-invariant submanifold of a GK-manifold $\bar{M}$. The distribution $D^{\perp} \oplus \xi$ is involutive if and only $A_{\varphi U} V=A_{\varphi V} U$ for $U, V \in \Gamma\left(D^{\perp}\right)$.

\section{References}

[1] Kenmotsu, K., “A class of almost contact Riemannian manifolds”, Tohoku Math. J. II Ser., 24 (1972) : 93-103.

[2] Pitiş G., "Geometry of Kenmotsu manifolds”, Publishing House of Transilvania University of Braşov, Braşov (2007).

[3] Falcitelli, M., Pastore, A. M., “f-structures of Kenmotsu type”, Mediterr J. Math., 3(3-4) (2006) : 549-564.

[4] Turgut Vanli, A., Sari, R., “Generalized Kenmotsu manifolds”, Communications in Mathematics and Applications, 7 4) (2016) : 311-328.

[5] Turgut Vanli, A., Sari, R., “On semi-invariant submanifolds of a Generalized Kenmotsu manifold admitting a semi-symmetric non-metric connection”, Pure and Applied Mathematics Journal, 4(1-2) (2015) : 14-18.

[6] Turgut Vanli, A., Sari, R., "On semi-invariant submanifolds of a generalized Kenmotsu manifold admitting a semi-symmetric metric connection”, Acta Universitatis Apulensis, 43 (2015) : 79-92.

[7] Ünal, İ., Sarı, R., Turgut Vanl1, A., “Concircular curvature tensor on generalized Kenmotsu manifolds”, Gümüşhane Üniversitesi Fen Bilimleri Enstitüsü Dergisi, CMES 2018 Ek Say1s1, (2018) : 99-105.

[8] Bagewadi, C. S., Venkatesha, S., "A study of distributions $\mathcal{L}$ and $\mathscr{M}$ of generalized Kenmotsu manifold”, Asian Research Journal of Mathematics, 8(4) (2018) : 1-10.

[9] Bejancu, A., "CR-submanifolds of a Kaehler manifold I”, Proc. Am. Math. Soc., 69 (1978) : 135-142.

[10] Bejancu, A., Papaghiuc, N., "Almost semi invariant submanifolds of a Sasakian manifold”, Bull. Math. Soc. Sci., 28 (1984) : 13-30.

[11] Ronsse, G. S., "Generic and skew CR-submanifolds of a Kaehler manifold”, Bull. Inst.Math. Acad. Sinica, 18 (1990) : 127-141.

[12] Siddiqi, M. D., Haseeb, A., Ahmad, M., "Skew semi-invariant submanifolds of generalized quasi-Sasakian manifolds”, Carpathian Mathematical Publications, 9(2), (2017) : 188-197. 
[13] Siddiqi, M. D., “On skew semi-invariant submanifolds of cosymplectic manifolds”, Asian Research Journal of Mathematics, (2018) : 1-11.

[14] Uddin, S., Kon, S. H., Khan, M. A., Singh, K., "Warped product semi-invariant submanifolds of nearly cosymplectic manifolds”, Mathematical Problems in Engineering, vol. 2011 Article ID 230374 (2011) : 12 pages.

[15] Sarı, R, Ünal, İ., Aksoy Sarı, E., "Skew semi invariant submanifolds of Para Kenmotsu manifold”, Gümüşhane Üniversitesi Fen Bilimleri Enstitüsü Dergisi , CMES 2018 Ek Say1s1 , (2018) : 112-118 .

[16] Goldberg, S. I., Yano, K., “Globally framed f-manifolds”, III. J. MAth.15 (1971) : 456474. 